\title{
More classes of non-orbit-transitive operators
}

\author{
by \\ Carl Pearcy and Lidia Smith (College Station, TX)
}

\begin{abstract}
In [9] and its sequel [7] the authors initiated a program whose (announced) goal is to eventually show that no operator in $\mathcal{L}(\mathcal{H})$ is orbit-transitive. In 9 it is shown, for example, that if $T \in \mathcal{L}(\mathcal{H})$ and the essential (Calkin) norm of $T$ is equal to its essential spectral radius, then no compact perturbation of $T$ is orbit-transitive, and in [7] this result is extended to say that no element of this same class of operators is weakly orbit-transitive. In the present note we show that no compact perturbation of certain 2-normal operators (which in general satisfy $\|T\|_{e}>r_{e}(T)$ ) can be orbit-transitive. This answers a question raised in 9]. Our main result herein is that if $T$ belongs to a certain class of 2-normal operators in $\mathcal{L}\left(\mathcal{H}^{(2)}\right)$ and there exist two constants $\delta, \rho>0$ satisfying $\left\|T^{k}\right\|_{e}>\rho k^{\delta}$ for all $k \in \mathbb{N}$, then for every compact operator $K$, the operator $T+K$ is not orbit-transitive. This seems to be the first result that yields non-orbit-transitive operators in which such a modest growth rate on $\left\|T^{k}\right\|_{e}$ is sufficient to give an orbit $\left\{T^{k} x\right\}$ tending to infinity.
\end{abstract}

1. Introduction. Let $\mathcal{H}$ be a separable, infinite-dimensional, complex Hilbert space, and denote the algebra of all bounded linear operators on $\mathcal{H}$ by $\mathcal{L}(\mathcal{H})$. If $T \in \mathcal{L}(\mathcal{H})$ and $x \in \mathcal{H}$, the countable (finite or infinite) set $\left\{T^{n} x\right\}_{n=0}^{\infty}$ is called the orbit of $x$ under $T$, and is denoted by $\mathcal{O}(x, T)$. If $\mathcal{O}(x, T)$ is dense in $\mathcal{H}$, then $x$ is called a hypercyclic vector for $T$, and $T$ is said to be a hypercyclic operator. The question of which operators in $\mathcal{L}(\mathcal{H})$ are hypercyclic and properties of the set of hypercyclic vectors of a hypercyclic operator have been much studied during the past twenty years. An operator $T$ in $\mathcal{L}(\mathcal{H})$ is called transitive if $T$ has no invariant subspace (closed linear manifold) other than $\{0\}$ and $\mathcal{H}$, and is called orbittransitive (or hypertransitive as in [9]) if every nonzero vector in $\mathcal{H}$ is hypercyclic for $T$. Presently one does not know whether there exist transitive or orbit-transitive operators in $\mathcal{L}(\mathcal{H})$. (It is obvious that every orbittransitive operator is transitive, and Read [13] has constructed an operator on the Banach space $\left(l_{1}\right)$ that is orbit-transitive.) Denote the set of all nontransitive operators in $\mathcal{L}(\mathcal{H})$ by $(\mathrm{NT})$ and the set of all non-orbittransitive operators in $\mathcal{L}(\mathcal{H})$ by $(\mathrm{NOT})$. The invariant subspace problem is

2010 Mathematics Subject Classification: 47A15, 47A16.

Key words and phrases: hypercyclic, orbit-transitive, hypertransitive. 
the open question whether $(\mathrm{NT})=\mathcal{L}(\mathcal{H})$, and the orbit-transitive operator problem is the question whether $(\mathrm{NOT})=\mathcal{L}(\mathcal{H})$. (The orbit-transitive operator problem is sometimes referred to as the hypertransitive-operator problem [9] or the nontrivial-invariant-closed-set problem. At present, neither the term "hypertransitive" nor "orbit-transitive" has been in use long enough to be considered standard, but note that if an operator $T$ in $\mathcal{L}(\mathcal{H})$ is called "supertransitive" if every nonzero vector $y$ in $\mathcal{H}$ is supercyclic for $T$, i.e., $\left\{\rho T^{n} y: n \in \mathbb{N}, \rho \in \mathbb{C}\right\}^{-}=\mathcal{H}$, then "hypertransitive" would seem to be a reasonable alternative to "orbit-transitive".)

As the abstract indicates, this paper is a continuation of the study, initiated in [9] and continued in [7], of classes of non-orbit-transitive operators, with the purpose (as mentioned explicitly in [9]) of eventually showing that $(\mathrm{NOT})=\mathcal{L}(\mathcal{H})$, and thus to give convincing evidence that operators on Hilbert space are very different creatures from operators on more general complex Banach spaces. More exactly, in this article we make progress on the problem of showing that if $T$ is $n$-normal and $K$ is compact, then $T+K \in$ (NOT). In particular, we produce the only known subset of (NOT) invariant under compact perturbations, consisting of operators which satisfy the very modest growth condition $\left\|T^{k}\right\|_{e} \geq \rho k^{\delta}$ for some $\rho, \delta>0$ and all $k \in \mathbb{N}$.

All the notation and terminology to follow is consistent with that of [9] and [7, but for the reader's convenience, we briefly review the main points. The sets of positive and nonnegative integers will be denoted by $\mathbb{N}$ and $\mathbb{N}_{0}$, and the complex plane by $\mathbb{C}$. The ideal of compact operators in $\mathcal{L}(\mathcal{H})$ will be written as $\mathbb{K}(\mathcal{H})$, or more simply as $\mathbb{K}$, and the quotient (Calkin) map $\mathcal{L}(\mathcal{H}) \rightarrow \mathcal{L}(\mathcal{H}) / \mathbb{K}$ by $\pi$. For $T$ in $\mathcal{L}(\mathcal{H})$ we write $\sigma(T)$ and $\sigma_{p}(T)$ for the spectrum and point spectrum of $T$, respectively, and $\sigma_{e}(T):=\sigma(\pi(T))$, $\sigma_{l r e}(T):=\sigma_{l r}(\pi(T))$ (the intersection of the left and right spectra of $\pi(T)$ ). We also write $r(T)$ and $r_{e}(T)$ for the spectral radii of $T$ and $\pi(T)$, as well as $\|T\|_{e}:=\|\pi(T)\|$.

Finally, for any positive integer $n$ we write $\mathcal{H}^{(n)}$ for the direct sum of $n$ copies of $\mathcal{H}$.

2. Some new ideas. Our first new result is a supplement to the following theorem of Ansari.

Theorem 2.1 (Ansari [1]). For every $T \in \mathcal{L}(\mathcal{H})$ and for every $n \in \mathbb{N}$, $T$ and $T^{n}$ have exactly the same set of hypercyclic vectors.

Our supplement is as follows:

Proposition 2.2. Suppose $T \in \mathcal{L}(\mathcal{H}), x \in \mathcal{H}$, and there exists $n_{0} \in \mathbb{N}$ such that $\left\|\left(T^{n_{0}}\right)^{k} x\right\| \stackrel{k}{\rightarrow} \infty$. Then $\left\|T^{k} x\right\| \stackrel{k}{\rightarrow} \infty$ too. Consequently,

$$
\left\{y \in \mathcal{H}:\left\|T^{k} y\right\| \stackrel{k}{\rightarrow} \infty\right\}=\left\{y \in \mathcal{H}: \exists n_{0} \in \mathbb{N} \text { with }\left\|\left(T^{n_{0}}\right)^{k} y\right\| \stackrel{k}{\rightarrow} \infty\right\} .
$$


Proof. Assume that $\left\|T^{k} x\right\| \nrightarrow \infty$. Then there exist $M \geq 0$ and a subsequence $\left\{k_{j}\right\} \subset \mathbb{N}$ such that

$$
\left\|T^{k_{j}} x\right\| \rightarrow M
$$

If, for $k_{j}>n_{0}$, we write $k_{j}=n_{0} q_{j}-r_{j}$, where $q_{j}, r_{j} \in \mathbb{N}_{0}, 0 \leq r_{j}<n_{0}$, we have

$$
\left\|\left(T^{n_{0}}\right)^{q_{j}} x\right\|=\left\|T^{r_{j}+k_{j}} x\right\| \leq\|T\|^{r_{j}} \cdot\left\|T^{k_{j}} x\right\| .
$$

But under the assumption 2.1 , 2.2 contradicts $\left\|\left(T^{n_{0}}\right)^{k} x\right\| \stackrel{k}{\rightarrow} \infty$.

The essence of the main new technique of this note is contained in the following proposition.

Proposition 2.3. Let $\left\{T_{n}\right\}_{n \in \mathbb{N}}$ be a sequence of operators in $\mathcal{L}(\mathcal{H})$ with the property that there exists a sequence of infinite-dimensional subspaces $\left\{\mathfrak{M}_{n}\right\}_{n \in \mathbb{N}}$ such that, for every $n \in \mathbb{N}$, the operator $T_{n}$ is bounded below on the subspaces $\mathfrak{M}_{n}, \ldots, \mathfrak{M}_{2 n-1}$ by some $M(n)>0$. Moreover, let $\left\{\alpha_{n}\right\}_{n \in \mathbb{N}}$ be any sequence in $l_{2}(\mathbb{N})$. Then for every $x_{0} \in \mathcal{H}$ there exists $y \in \mathcal{H}$ such that

$$
\left\|y-x_{0}\right\|^{2} \leq \sum_{i=1}^{\infty}\left|\alpha_{i}\right|^{2}, \quad\left\|T_{n} y\right\|^{2} \geq \sum_{i=n}^{2 n-1}\left|\alpha_{i}\right|^{2} M^{2}(n), \quad n \in \mathbb{N} .
$$

Proof. From each subspace $\mathfrak{M}_{n}$ we will choose by induction a unit vector $f_{n}$ such that the sequence $\left\{f_{i}\right\}_{i=1}^{\infty}$ will satisfy a set of orthogonality conditions, and we will define

$$
y=x_{0}+\sum_{i=1}^{\infty} \alpha_{i} f_{i}
$$

To simplify notation, let $f_{0}:=x_{0}, \alpha_{0}:=1, T_{0}:=I$, and

$$
y_{n}=\sum_{i=0}^{n} \alpha_{i} f_{i}, \quad n \in \mathbb{N} .
$$

We will choose the vector $f_{n} \in \mathfrak{M}_{n}$, with $\left\|f_{n}\right\|=1$, such that

$$
T_{k} f_{n} \perp T_{k} f_{j}, \quad 0 \leq j \leq n-1,0 \leq k \leq n .
$$

After defining $f_{j}$ we will have, by hypothesis, for every $n \in \mathbb{N}$,

$$
\left\|T_{n} f_{j}\right\| \geq M(n), \quad n \leq j \leq 2 n-1,
$$

and for $n \leq i, j, i \neq j$, we will have $T_{n} f_{i} \perp T_{n} y_{n-1}$ and $T_{n} f_{j} \perp T_{n} f_{i}$, so

$$
\begin{aligned}
\left\|T_{n} y\right\|^{2} & =\left\|T_{n}\left(y_{n-1}+\sum_{i=n}^{\infty} \alpha_{i} f_{i}\right)\right\|^{2}=\left\|T_{n} y_{n-1}\right\|^{2}+\left\|T_{n}\left(\sum_{i=n}^{\infty} \alpha_{i} f_{i}\right)\right\|^{2} \\
& =\left\|T_{n} y_{n-1}\right\|^{2}+\sum_{i=n}^{\infty}\left|\alpha_{i}\right|^{2}\left\|T_{n} f_{i}\right\|^{2}
\end{aligned}
$$


Now we give more details on the recursive construction of the sequence $\left\{f_{n}\right\}$. If $\mathfrak{M}$ is a subspace of $\mathcal{H}$, we will write $\mathcal{P}_{\mathfrak{M}}$ for the (orthogonal) projection of $\mathcal{H}$ onto $\mathfrak{M}$.

Choose $f_{1} \in \mathfrak{M}_{1}$ such that $\left\|f_{1}\right\|=1, f_{1} \perp f_{0}$ and $T_{1} f_{1} \perp T_{1} f_{0}$. To accomplish this, define $\mathcal{S}_{1}=\bigvee\left\{f_{0}, T_{1}^{*} T_{1} f_{0}\right\}$ and choose $f_{1} \in \mathfrak{M}_{1} \ominus \mathcal{P}_{\mathfrak{M}_{1}}\left(\mathcal{S}_{1}\right)$.

In general, in order to have all the conditions in (2.4) satisfied, after $f_{0}, \ldots, f_{n-1}$ have been defined, set $\mathcal{S}_{n}=\bigvee_{0 \leq j \leq n-1,0 \leq k \leq n}\left\{T_{k}^{*} T_{k} f_{j}\right\}$, which is a finite-dimensional vector space, and choose $f_{n}$ to be a unit vector in $\mathfrak{M}_{n} \ominus \mathcal{P}_{\mathfrak{M}_{n}}\left(\mathcal{S}_{n}\right)$. This defines the sequence $\left\{f_{n}\right\}_{n \in \mathbb{N}_{0}}$ with the desired properties.

Using (2.5) and (2.6) we have

$$
\left\|T_{n} y\right\|^{2} \geq \sum_{i=n}^{\infty}\left|\alpha_{i}\right|^{2}\left\|T_{n} f_{i}\right\|^{2} \geq \sum_{i=n}^{2 n-1}\left|\alpha_{i}\right|^{2} M^{2}(n), \quad n \in \mathbb{N} .
$$

REMARK 2.4. Notice first that the hypothesis of Proposition 2.3 could be modified so as to be valid only for $n$ sufficiently large without changing the conclusion. Moreover, the relation between the sequence of operators $\left\{T_{n}\right\}$ and the sequence of subspaces $\left\{\mathfrak{M}_{n}\right\}$ has an essentially equivalent formulation, as follows: given the sequence of operators $\left\{T_{n}\right\}$, there exist a sequence of infinite-dimensional subspaces $\left\{\mathfrak{M}_{n}\right\}$ and a sequence of positive real numbers $\{N(n)\}$ such that, for every $n$ sufficiently large, all operators from the set $T_{[(n+1) / 2]}, \ldots, T_{n}$ are bounded below by $N(n)$ on $\mathfrak{M}_{n}$.

The following is one of our two main results.

THEOREM 2.5. Let $\left\{T_{n}\right\}_{n \in \mathbb{N}} \subset \mathcal{L}(\mathcal{H})$ be such that there exist positive numbers $\rho, \delta$ with the property that for every $n \in \mathbb{N}$ sufficiently large, there exists an infinite-dimensional subspace $\mathfrak{M}_{n}$ on which $T_{[(n+1) / 2]}, \ldots, T_{n}$ are bounded below by $\rho n^{\delta}$, and let $\left\{K_{n}\right\}$ be any sequence of compact operators. Define

$$
A_{n}=T_{n}+K_{n}, \quad n \in \mathbb{N} .
$$

Then the set of vectors $y \in \mathcal{H}$ such that $\left\|A_{n} y\right\| \rightarrow \infty$ is dense in $\mathcal{H}$.

Proof. Fix $\varepsilon$ such that $0<\varepsilon<1$ and choose $N_{0}$ sufficiently large that, for $n \geq N_{0}$, there exists an infinite-dimensional subspace $\mathfrak{M}_{n}$ on which $T_{[(n+1) / 2]}, \ldots, T_{n}$ are bounded below by $\rho n^{\delta}$. Note that the compact operators $K_{[(n+1) / 2]}, \ldots, K_{n}$, when restricted to $\mathfrak{M}_{n}$, remain compact operators, and thus for $j=[(n+1) / 2], \ldots, n$, there is a finite-dimensional subspace $\mathfrak{F}_{j} \subset \mathfrak{M}_{n}$ such that $\left\|K_{j} \mid\left(\mathfrak{M}_{n} \ominus \mathfrak{F}_{j}\right)\right\|<\varepsilon \rho n^{\delta}$. Thus $\left\|K_{j} \mid\left(\mathfrak{M}_{n} \ominus \bigvee_{j=[(n+1) / 2]}^{n} \mathfrak{F}_{j}\right)\right\|<\varepsilon \rho n^{\delta}$ for $j=[(n+1) / 2], \ldots, n$. Thus by defining $\mathfrak{N}_{n}=\mathfrak{M}_{n} \ominus\left(\bigvee_{j=[(n+1) / 2]}^{n} \mathfrak{F}_{j}\right)$, we see that each of the operators $A_{[(n+1) / 2]}, \ldots, A_{n}$ is bounded below on $\mathfrak{N}_{n}$ by $N(n):=(1-\varepsilon) \rho n^{\delta}$. 
Next, note that the sequence of operators $\left\{A_{n}\right\}$, together with the sequence of subspaces $\left\{\mathfrak{N}_{n}\right\}$ and the sequence of lower bounds $\{N(n)\}$, satisfies the hypothesis of Proposition 2.3 (and Remark 2.4). Given an arbitrary vector $x_{0} \in \mathcal{H}$, let $y$ be as in the conclusion of Proposition 2.3. Then we have

$$
\left\|y-x_{0}\right\|^{2} \leq \sum_{i=1}^{\infty}\left|\alpha_{i}\right|^{2}, \quad\left\|A_{n} y\right\|^{2} \geq \sum_{i=n}^{2 n-1}\left|\alpha_{i}\right|^{2} N^{2}(n), \quad n \in \mathbb{N} .
$$

Take $\alpha_{n}=\varepsilon / n^{(1+\delta) / 2}$. Then

$$
\begin{aligned}
\left\|A_{n} y\right\|^{2} & \geq(1-\varepsilon)^{2} \rho^{2} \sum_{i=n}^{2 n-1} \frac{\varepsilon^{2}}{i^{1+\delta}} \cdot n^{2 \delta} \\
& \geq(1-\varepsilon)^{2} \frac{\rho^{2}}{2^{1+\delta}} \cdot \sum_{i=n}^{2 n-1} \frac{\varepsilon^{2}}{n^{1-\delta}}=(1-\varepsilon)^{2} \frac{\rho^{2} \varepsilon^{2}}{2^{1+\delta}} \cdot n^{\delta},
\end{aligned}
$$

and

$$
\left\|y-x_{0}\right\|^{2} \leq \varepsilon^{2} \sum_{i=1}^{\infty} \frac{1}{i^{1+\delta}}
$$

from which it is immediate that $\left\|A_{n} y\right\| \rightarrow \infty$ and that the set of vectors $y$ with this property is dense in $\mathcal{H}$.

The obvious application to powers of a single operator is this:

Corollary 2.6. Assume $T \in \mathcal{L}(\mathcal{H})$ has the property that there exist positive numbers $\rho, \delta$ such that for every $n \in \mathbb{N}$ sufficiently large, there exists an infinite-dimensional subspace $\mathfrak{M}_{n}$ on which the powers $T^{[(n+1) / 2]}, \ldots, T^{n}$ are bounded below by $\rho n^{\delta}$. If $K \in \mathbb{K}$ and $A:=T+K$, then there exists a dense set of vectors $y$ in $\mathcal{H}$ such that $\left\|A^{n} y\right\| \rightarrow \infty$.

Proof. There exists a sequence of compact operators $\left\{K_{n}\right\}$ such that

$$
A^{n}=T^{n}+K_{n}
$$

Theorem 2.5 has a generalization that should be quite useful in enlarging the class $(\mathrm{NOT})$.

TheOREM 2.7. Suppose that $\left\{B_{n}\right\} \in \mathcal{L}\left(\mathcal{H}^{(2)}\right)$ has the property that

$$
B_{n}=\left(\begin{array}{cc}
T_{n} & * \\
0 & *
\end{array}\right) \text {, }
$$

where the asterisks denote arbitrary entries, and $\left\{T_{n}\right\}_{n \in \mathbb{N}} \subset \mathcal{L}(\mathcal{H})$ is such that there exist positive numbers $\rho, \delta$ with the property that for every $n \in \mathbb{N}$ sufficiently large, there exists an infinite-dimensional subspace $\mathfrak{M}_{n}$ on which $T_{[(n+1) / 2]}, \ldots, T_{n}$ are bounded below by $\rho n^{\delta}$. Let $\left\{K_{n}\right\} \subset \mathbb{K}\left(\mathcal{H}^{(2)}\right)$ and define $A_{n}=B_{n}+K_{n}$. Then the set of vectors $y \in \mathcal{H}^{(2)}$ satisfying $\left\|A_{n} y\right\| \rightarrow \infty$ is dense in $\mathcal{H} \oplus(0)$. 
Proof. It is clear that the sequence of operators $\left\{B_{n}\right\}$ together with the sequence of subspaces $\left\{\mathfrak{M}_{n} \oplus(0)\right\}$ satisfies the hypothesis of Theorem 2.5, and thus the conclusion is immediate.

We turn now to the application of the results of Section 2 to the class of $n$-normal operators.

3. $n$-Normal operators. Recall that for any $n \in \mathbb{N}$, an operator $T \in$ $\mathcal{L}(\mathcal{H})$ is called an $n$-normal operator if $T$ is unitarily equivalent to an $n \times n$ operator matrix $\left(N_{i j}\right)$ acting on $\mathcal{H}^{(n)}$ in the usual fashion, where the set $\left\{N_{i j}\right\}$ consists of mutually commuting normal operators in $\mathcal{L}(\mathcal{H})$. The theory of $n$ normal operators is quite well developed. It was begun in [3], and continued in [10], [1], [5], [6] and [8], where it was proved that every $n$-normal operator has a nontrivial hyperinvariant subspace (n.h.s.).

We will show below that compact perturbations of certain classes of 2normal operators are subsets of (NOT) by virtue of having an orbit that tends to infinity.

We begin with the following well-known fact from [5].

Proposition 3.1. Let $T$ be any $n$-normal operator in $\mathcal{L}(\mathcal{H})$. Then $T$ is unitarily equivalent to an n-normal operator $\left(N_{i j}\right)$, acting on $\mathcal{H}^{(n)}$ in the usual matricial fashion, which satisfies

(i) the $N_{i j}$ are mutually commuting normal operators in $\mathcal{L}(\mathcal{H})$,

(ii) $N_{i j}=0$ whenever $i>j$, i.e., the matrix $\left(N_{i j}\right)$ is in upper triangular form.

Notation 3.2. We shall say that an $n$-normal operator is in standard form if it is an $n \times n$ matrix $\left(N_{i j}\right)$ acting as usual on $\mathcal{H}^{(n)}$ and satisfying (i) and (ii) of Proposition 3.1. (Of course, except in rare cases the standard form of an $n$-normal operator is not unique.)

The next lemma is elementary.

Lemma 3.3. Let $T=\left(N_{i j}\right) \in \mathcal{L}\left(\mathcal{H}^{(2)}\right)$ be a 2-normal operator in standard form, and suppose that the polar decompositions $N_{11}=V_{1} P_{1}$ and $N_{22}=V_{2} P_{2}$ (with $P_{1}, P_{2} \geq 0$ ) satisfy $V_{1}=V_{2}$. Then there exists a unitary operator $U \in \mathcal{L}(\mathcal{H})$ such that $N_{i i}=U P_{i}, i=1,2$, and $U$ commutes with all the $N_{i j}$. Moreover, for every $K \in \mathbb{K}$, there exists a sequence $\left\{J_{k}\right\}_{k \in \mathbb{N}} \subset \mathbb{K}$ such that

$$
\left\|(T+K)^{k} x\right\|=\left\|\left(S^{k}+J_{k}\right) x\right\|, \quad k \in \mathbb{N}, x \in \mathcal{H},
$$

where $S$ is the 2-normal operator in $\mathcal{L}\left(\mathcal{H}^{(2)}\right)$ in standard form given by the matrix

$$
S=\left(\begin{array}{cc}
P_{1} & U^{-1} N_{12} \\
0 & P_{2}
\end{array}\right)
$$


Proof. The first statement of the lemma is an easy consequence of the spectral theorem for normal operators. Next, write $\operatorname{Diag}(U)$ for the $2 \times 2$ diagonal matrix with each diagonal entry equal to $U$. Note that $S$ and $\operatorname{Diag}(U)$ are mutually commuting 2-normal operators in standard form, and $T=\operatorname{Diag}(U) S$. Furthermore, if we write $(T+K)^{k}=T^{k}+K_{k}$ where $K_{k} \in \mathbb{K}$, we obtain

$$
\begin{aligned}
(T+K)^{k} & =T^{k}+K_{k}=(\operatorname{Diag}(U) S)^{k}+K_{k} \\
& =\operatorname{Diag}\left(U^{k}\right)\left(S^{k}+J_{k}\right), \quad k \in \mathbb{N},
\end{aligned}
$$

where $J_{k}:=\operatorname{Diag}\left(U^{-k}\right) K_{k} \in \mathbb{K}$, and since for each $k \in \mathbb{N}$, $\operatorname{Diag}\left(U^{k}\right)$ is a unitary operator, (3.1) is immediate from (3.3).

We turn now to some preliminary lemmas.

LEMmA 3.4. Let $1 \geq a \geq b \geq 0$ and let $k$ be a given positive integer. Then, for every $m \in \mathbb{N} \cap[k / 2, k]$, we have

$$
a^{m}-b^{m} \geq \frac{1}{2}\left(a^{k}-b^{k}\right),
$$

and thus

$$
\sum_{i=0}^{m-1} a^{m-1-i} b^{i} \geq \frac{1}{2} \sum_{i=0}^{k-1} a^{k-1-i} b^{i} .
$$

Proof. The inequality (3.4) is equivalent to

$$
b^{k}-2 b^{m} \geq a^{k}-2 a^{m} \quad \text { for } k / 2 \leq m \leq k .
$$

Consider the function $f(x)=x^{k}-2 x^{m}$. Then $f^{\prime}(x)=k x^{k-1}-2 m x^{m-1}=$ $x^{m-1}\left(k x^{k-m}-2 m\right)$. But since $2 m \geq k$ we have $f^{\prime}(x) \leq x^{m-1}\left(k x^{k-m}-k\right)=$ $x^{m-1} k\left(x^{k-m}-1\right)$, and for $x \in[0,1]$ we have $f^{\prime}(x) \leq 0$. Thus $f$ is decreasing on the interval $[0,1]$ and thus (3.4) follows.

The inequality (3.5) is immediate in the case $a=b$, and it follows from (3.4) by dividing by $a-b$ when $a>b$.

LeMma 3.5. Suppose

$$
T=\left(\begin{array}{cc}
P_{1} & N \\
0 & P_{2}
\end{array}\right)
$$

is a 2-normal operator in standard form, where $P_{i} \geq 0, i=1,2$, and let $K \in \mathbb{K}$ and $A=T+K$. Then

$$
T^{k}=\left(\begin{array}{cc}
P_{1}^{k} & N \sum_{i=0}^{k-1}\left(P_{1}^{k-1-i} P_{2}^{i}\right) \\
0 & P_{2}^{k}
\end{array}\right), \quad k \in \mathbb{N},
$$

and $A^{k}=T^{k}+K_{k}$, where $K_{k} \in \mathbb{K}$. Moreover, if $\max \left\{\left\|P_{1}\right\|_{e},\left\|P_{2}\right\|_{e}\right\}>1$, then there exists a vector $x \in \mathcal{H}^{(2)}$ such that $\left\|A^{k} x\right\| \rightarrow \infty$. 
Proof. Equation (3.6) results from an easy calculation, and since $r_{e}(A)=$ $\max \left\{\left\|P_{1}\right\|_{e},\left\|P_{2}\right\|_{e}\right\}$, if this maximum is greater than 1 , then the existence of such a vector $x$ is immediate from Corollary 1.6 of [9].

The next result is our second main theorem.

Theorem 3.6. Suppose

$$
T=\left(\begin{array}{cc}
N_{1} & M \\
0 & N_{2}
\end{array}\right)
$$

is a 2-normal operator in standard form, with $N_{1}=V P_{1}$ and $N_{2}=V P_{2}$ (polar decompositions), $K \in \mathbb{K}$, and there exist $\rho, \delta>0$ such that

$$
\left\|T^{k}\right\|_{e}>\rho k^{\delta}, \quad k \in \mathbb{N} .
$$

Then there exists $x \in \mathcal{H}^{(2)}$ such that $\left\|(T+K)^{n} x\right\| \rightarrow \infty$, and consequently $T+K \in(\mathrm{NOT})$.

Proof. By Lemmas 3.3 and 3.5, with

$$
S=\left(\begin{array}{cc}
P_{1} & N \\
0 & P_{2}
\end{array}\right)
$$

a 2-normal operator in standard form, it suffices to show that if $\left\{J_{k}\right\}$ is any sequence of compact operators, then there exists $x \in \mathcal{H}^{(2)}$ such that

$$
\left\|\left(S^{k}+J_{k}\right) x\right\| \rightarrow \infty \text {. }
$$

Moreover, we may suppose that $\left\|P_{1}\right\|_{e},\left\|P_{2}\right\|_{e} \leq 1$, and that (via (3.3)

$$
\left\|S^{k}\right\|_{e}=\left\|T^{k}\right\|_{e} \geq \rho k^{\delta}, \quad k \in \mathbb{N} .
$$

Applying (3.9), 3.8) and Lemma 3.5, we immediately obtain

$$
\left\|N \sum_{i=0}^{k-1} P_{1}^{k-1-i} P_{2}^{i}\right\|_{e}>\rho k^{\delta}-1>\hat{\rho} k^{\delta},
$$

where $\hat{\rho}>0$ is defined appropriately for $k$ sufficiently large.

Since $P_{1}, P_{2}$ and $N$ are mutually commuting normal operators, it follows from [4] that there exist compact operators $J_{1}, J_{2}, J_{3}$, an orthonormal basis $\mathcal{E}=\left\{e_{n}\right\}_{n \in \mathbb{N}}$ for $\mathcal{H}$, and sequences $\left\{\alpha_{n}\right\},\left\{\beta_{n}\right\}$ and $\left\{\gamma_{n}\right\}$ in $\left(l_{\infty}\right)$, with $0 \leq$ $\alpha_{n}, \beta_{n} \leq 1$, such that

$$
P_{1}=\operatorname{Diag}\left(\alpha_{n}\right)+J_{1}, \quad P_{2}=\operatorname{Diag}\left(\beta_{n}\right)+J_{2}, \quad N=\operatorname{Diag}\left(\gamma_{n}\right)+J_{3},
$$

and thus (see 3.10$)$ ) the $(1,2)$ entry of $S^{k}$ is

$$
\operatorname{Diag}\left(\gamma_{n} \sum_{i=0}^{k-1}\left(\alpha_{n}^{k-1-i} \beta_{n}^{i}\right)\right)+J_{4}^{(k)}, \quad k \in \mathbb{N},
$$


where $J_{4}^{(k)}$ is compact. Moreover, since

$$
\left\|N \sum_{i=0}^{k-1} P_{1}^{k-1-i} P_{2}^{i}\right\|_{e}=r_{e}\left(\operatorname{Diag}\left(\gamma_{n} \sum_{i=0}^{k-1}\left(\alpha_{n}^{k-1-i} \beta_{n}^{i}\right)\right)\right)
$$

is a largest (in modulus) limit point of the sequence

$$
\left\{\gamma_{n} \sum_{i=0}^{k-1} \alpha_{n}^{k-1-i} \beta_{n}^{i}\right\}
$$

for each fixed $k \in \mathbb{N}$ there exists a subsequence

$$
\left\{\left|\gamma_{n_{q}}\right| \sum_{i=0}^{k-1} \alpha_{n_{q}}^{k-1-i} \beta_{n_{q}}^{i}\right\}_{q}
$$

where $\left\{n_{q}\right\}$ depends on $k$, that converges to $\left\|N \sum_{i=0}^{k-1} P_{1}^{k-1-i} P_{2}^{i}\right\|_{e}$. Thus for $n_{q}$ sufficiently large (say $q \geq q_{0}$ ), we see, with

$$
\mathfrak{M}_{k}=\bigvee\left\{e_{n_{q}}\right\}_{q \geq q_{0}},
$$

that $N \sum_{i=0}^{k-1} P_{1}^{k-1-i} P_{2}^{i}$ is bounded below on $\mathfrak{M}_{k}$ by $\hat{\rho} k^{\delta}$ (see 3.10 ), and thus $S^{k}$ has the same lower bound on $(0) \oplus \mathfrak{M}_{k}$.

By Lemma 3.4, for $m=[(k+1) / 2], \ldots, k$ we have

$$
\left|\gamma_{n}\right| \sum_{i=0}^{m-1} \alpha_{n}^{m-1-i} \beta_{n}^{i} \geq \frac{1}{2}\left(\left|\gamma_{n}\right| \sum_{i=0}^{k-1} \alpha_{n}^{k-1-i} \beta_{n}^{i}\right),
$$

and using this fact for the subsequence of indices $\left\{n_{q}\right\}$ we deduce that $S^{[(k+1) / 2]}, \ldots, S^{k}$ have $\frac{1}{2} \hat{\rho} k^{\delta}$ as a lower bound on $(0) \oplus \mathfrak{M}_{k}$. Now the conclusion follows from Theorem 2.5.

This result combined with Theorem 2.7 yields the following:

COROLlary 3.7. If $R$ is an operator that has an invariant subspace on which it is a 2-normal operator satisfying the hypothesis of Theorem 3.6, and $A=R+K$, where $K$ is compact, then $A$ has an orbit tending to infinity.

REMARK 3.8. It follows as in Theorem 2.5 that the set of vectors $x$ as in the conclusion of Theorem 3.6 is dense in $\mathcal{H}$.

We continue by recalling the definition of the operator $A$ from Example 4.5 of [9]. (The question whether $A$ belongs to (NOT) was left unresolved there.)

EXAMPLE 3.9. Let $H$ be a Hermitian 2-normal operator in $\mathcal{L}\left(\mathcal{H}^{(2)}\right)$ represented as an operator matrix

$$
H=\left(\begin{array}{cc}
H_{1} & 0 \\
0 & H_{2}
\end{array}\right)
$$


where $H_{1}$ and $H_{2}$ are commuting Hermitian operators in $\mathcal{L}(\mathcal{H})$ satisfying $\sigma\left(H_{i}\right)=\sigma_{e}\left(H_{i}\right)=[1 / 2,1], i=1,2$, and on the space $\bigoplus_{n=2}^{\infty} \mathcal{H}^{(2)}$ consider the 2-normal operator

$$
T=\bigoplus_{n=2}^{\infty}\left(\begin{array}{cc}
(1-1 / n) 1_{\mathcal{H}} & (1 / \sqrt{n}) 1_{\mathcal{H}} \\
0 & (1-1 / n) 1_{\mathcal{H}}
\end{array}\right) .
$$

Then, as was noted in [9], for all $k \in \mathbb{N},\left\|T^{k}\right\|_{e}$ satisfies

$$
\frac{k}{\sqrt{2 k-1}}\left(\frac{2 k-2}{2 k-1}\right)^{k-1} \leq\left\|T^{k}\right\|_{e} \leq 1+\frac{k}{\sqrt{2 k-1}}\left(\frac{2 k-2}{2 k-1}\right)^{k-1} \text {. }
$$

Let $K$ be an arbitrary compact operator on $\bigoplus_{n=1}^{\infty} \mathcal{H}^{(2)}$ and set

$$
A=(H \oplus T)+K \text {. }
$$

(The presence here of the direct summand $H$ is simply to prevent $A$ from having disconnected spectrum, and thus to have a nontrivial hyperinvariant subspace.) Then $\sigma_{e}(A)=\sigma_{e}(H \oplus T)=[1 / 2,1]$, but $A$ is not essentially power bounded. In fact, from 3.12 we see that, asymptotically, $\left\|A^{k}\right\|_{e}=$ $\left\|T^{k}\right\|_{e} \sim \sqrt{k}$; and this growth is too slow for [9, Th. 1.5] to be applicable. However, that the operator $A$ in $(3.13)$ belongs to (NOT) is now immediate from Theorem 3.6.

A natural question to ask, in view of Theorem 3.6, is whether the hypothesis (3.7) is really necessary in order to conclude that $T$ has some orbit converging to infinity. Example 4.1 below shows that (3.7) cannot be omitted in general, but the next proposition shows that there are some classes of $n$-normal operators in which (3.7) is not needed.

Proposition 3.10. Suppose $K \in \mathbb{K}\left(\mathcal{H}^{(2)}\right)$ and

$$
T=\left(\begin{array}{cc}
N_{1} & N_{3} \\
0 & N_{2}
\end{array}\right) \in \mathcal{L}\left(\mathcal{H}^{(2)}\right)
$$

is a 2-normal operator in standard form such that $\max \left\{r_{e}\left(N_{1}\right), r_{e}\left(N_{2}\right)\right\}<1$. Then $T+K$ is essentially power bounded, and thus (by [9, Theorem 1.2]) belongs to (NOT). Moreover, if, on the other hand, $\sigma_{e}\left(N_{1}\right)=\sigma_{e}\left(N_{2}\right)=\{1\}$ and $N_{3}$ is essentially invertible (i.e., $\pi\left(N_{3}\right)$ is invertible), then either $T+K$ is essentially power bounded or $\left\|(T+K)^{k}\right\|_{e} \sim k$ asymptotically, so again (by [9, Theorems 1.2 and 1.5]), $T+K \in(\mathrm{NOT})$.

Proof. Since $r_{e}(T+K)=r_{e}(T)=\max \left\{r_{e}\left(N_{1}\right), r_{e}\left(N_{2}\right)\right\}$, if $r_{e}(T+K)<1$, then the general theory of Banach algebras implies that $\left\|(T+K)^{k}\right\|_{e}$ $\stackrel{k}{\rightarrow} 0$, so $T+K$ is essentially power bounded. Now suppose that $\sigma_{e}\left(N_{1}\right)=$ $\sigma_{e}\left(N_{2}\right)=\{1\}$ and $\pi\left(N_{3}\right)$ is invertible. Then one knows from [12] that there exist compact operators $K_{i}, i=1,2,3$, in $\mathcal{L}(\mathcal{H})$ such that $N_{1}=1_{\mathcal{H}}+K_{1}$, 
$N_{2}=1_{\mathcal{H}}+K_{2}$, and $N_{3}=J+K_{3}$, where $J$ is invertible. Thus

$$
T=\left(\begin{array}{cc}
1_{\mathcal{H}} & J \\
0 & 1_{\mathcal{H}}
\end{array}\right)+K,
$$

where $K \in \mathcal{L}\left(\mathcal{H}^{(2)}\right)$ is compact. An easy matricial calculation ([9, Prop. 3.2]) gives $\left\|(T+K)^{k}\right\|_{e}=\left\|T^{k}\right\|_{e} \sim k$, and it is now immediate from [9, Theorem 1.5] that $T+K$ has (a dense set of) orbits tending to infinity.

4. Oscillating behavior. Here we construct a 2-normal operator $B=$ $\left(B_{i j}\right) \in \mathcal{L}\left(\mathcal{H}^{(2)}\right)$ in standard form, with $B_{11}=B_{22} \geq 0$, but $B$ is not essentially power bounded, and neither does it satisfy the hypothesis of Theorem 3.6. The operator $B$ will have the property that there exists a subsequence of powers $\left\{k_{n}\right\}$ and $\rho, \delta>0$ such that $\left\|B^{k_{n}}\right\|_{e}>\rho k_{n}^{\delta}$ for all $k_{n}$, but at the same time, there exists a subsequence of powers $\left\{j_{n}\right\}$ such that $\left\{\left\|B^{j_{n}}\right\|\right\}$ is bounded.

EXAMPLE 4.1. Let

$$
B=\bigoplus_{n=2}^{\infty} T_{m_{n}}
$$

where $T_{n}$ is given by

$$
T_{n}:=\left(\begin{array}{cc}
(1-1 / n) 1_{\mathcal{H}} & (1 / \sqrt{n}) 1_{\mathcal{H}} \\
0 & (1-1 / n) 1_{\mathcal{H}}
\end{array}\right), \quad n \in \mathbb{N}
$$

and $\left\{m_{n}\right\}$ is defined recursively as $m_{2}=2, m_{n+1}=m_{n}^{4}$, which gives $m_{n}$ $=2^{4^{n-2}}$. The sequences of powers $\left\{k_{n}\right\}$ and $\left\{j_{n}\right\}$ are given by $k_{n}:=m_{n}$ and $j_{n}:=m_{n}^{2}$.

Note that

$$
T_{n}^{k}=\left(\begin{array}{cc}
(1-1 / n)^{k} 1_{\mathcal{H}} & (k / \sqrt{n})(1-1 / n)^{k} 1_{\mathcal{H}} \\
0 & (1-1 / n)^{k} 1_{\mathcal{H}}
\end{array}\right) .
$$

Consider the magnitude of the $(1,2)$ entry of $T_{n}^{k}$ as a function of $n$, with $k$ as parameter, denoted as

$$
g(n)=\frac{k}{\sqrt{n}}\left(1-\frac{1}{n}\right)^{k-1} .
$$

Note that the $(1,2)$ entry of $T_{n}^{k}$ is the one that determines the growth of the essential norm, since the diagonal entries $T_{n}^{k}$ have norms bounded by 1 . The first derivative of $g$ is

$$
g^{\prime}(n)=\frac{1}{n} \frac{k}{\sqrt{n}}\left(1-\frac{1}{n}\right)^{k-2}\left(-\frac{1}{2} \frac{n-1}{n}+\frac{k-1}{n}\right),
$$


with the only zero of interest at

$$
n=2 k-1 \text {. }
$$

By computing the second derivative we note that $n=2 k-1$ is a maximum point for $g$.

We are now ready to estimate the growth of the sequences $\left\{\left\|B^{k_{n}}\right\|_{e}\right\}$ and $\left\{\left\|B^{j_{n}}\right\|\right\}$. As noted above, the growth of $T_{m_{n}}^{k}$ is determined by the expression

$$
\frac{k}{\sqrt{m_{n}}}\left(1-\frac{1}{m_{n}}\right)^{k-1}
$$

For the first sequence, we observe that $\left\|B^{k_{n}}\right\|_{e}=\left\|B^{m_{n}}\right\|_{e} \geq\left\|T_{m_{n}}^{m_{n}}\right\|_{e}$ and since the sequence $\left\{\left(1-1 / m_{n}\right)^{m_{n}-1}\right\}_{n}$ is decreasing to $1 / e$, we have

$$
\left\|T_{m_{n}}^{m_{n}}\right\|_{e} \geq \sqrt{m_{n}}\left(1-\frac{1}{m_{n}}\right)^{m_{n}-1}>\sqrt{m_{n}} \cdot \frac{1}{e} .
$$

It follows that here we can take $\delta=1 / 2$ and $\rho=1 / 3$ to get

$$
\left\|B^{k_{n}}\right\|_{e}>\rho k_{n}^{\delta} \text {. }
$$

Next, to show that $\left\{\left\|B^{j_{n}}\right\|\right\}$ is a bounded sequence, it is sufficient to show that there exists a constant $M$ such that for all $n$ and $p$ we have

$$
\left\|T_{m_{p}}^{j_{n}}\right\| \leq M \text {. }
$$

By the above remarks, for the fixed power $k:=j_{n}=m_{n}^{2}$ (and thus for a fixed $n$ ), we deduce that the entry of $T_{q}^{k}$ that determines the growth of the $\left\|T_{q}^{k}\right\|$ has a maximum at $q_{0}=2 k-1\left(=2 m_{n}^{2}-1\right)$, increasing for $q$ up to that value and decreasing after the maximum is attained. Notice that the sequence $\left\{m_{r}\right\}_{r}$ does not assume the value $q_{0}$; in particular, $m_{n}<q_{0}<m_{n+1}$. Thus, to show $\left\|T_{m_{p}}^{j_{n}}\right\|$ is bounded for all $p$ and $n$, it is sufficient to show that, for fixed $n$, the norms $\left\|T_{m_{n}}^{j_{n}}\right\|$ and $\left\|T_{m_{n+1}}^{j_{n}}\right\|$ have upper bounds independent of $n$.

The norm of the $(1,2)$ entry of $T_{m_{n+1}}^{j_{n}}$ is

$$
\left(1-\frac{1}{m_{n}^{4}}\right)^{m_{n}^{2}-1} \leq 1
$$

and the norm of the $(1,2)$ entry of $T_{m_{n}}^{j_{n}}$ is

$$
\frac{m_{n}^{2}}{\sqrt{m_{n}}}\left(1-\frac{1}{m_{n}}\right)^{m_{n}^{2}-1}=2 \cdot m_{n}^{3 / 2}\left(1-\frac{1}{m_{n}}\right)^{m_{n}^{2}} \leq 2 \cdot m_{n}^{3 / 2}\left(\frac{1}{2}\right)^{m_{n}},
$$

since the sequence $\left\{\left(1-1 / m_{n}\right)^{m_{n}}\right\}_{n}$ is increasing and bounded by $1 / e$; thus $\left\|T_{m_{n}}^{j_{n}}\right\| \stackrel{n}{\rightarrow} 0$.

5. Open problems. We close with some open problems that seem to be of interest. 
Problem 5.1. If the operator $H$ is as in (3.11) and $B$ is the operator from Example 4.1, how does one show that a compact perturbation of $H \oplus B$ belongs to (NOT)?

Problem 5.2. Does every compact perturbation of an arbitrary $n$-normal operator belong to (NOT)?

\section{References}

[1] S. Ansari, Hypercyclic and cyclic vectors, J. Funct. Anal. 128 (1995), 374-383.

[2] B. Beauzamy, Introduction to Operator Theory and Invariant Subspaces, NorthHolland, 1988.

[3] A. Brown, Unitary equivalence of binormal operators, Amer. J. Math. 76 (1954), 414-434.

[4] L. Brown, R. G. Douglas and P. Fillmore, Extensions of $C^{*}$-algebras and $K$-homology, Ann. of Math. 105 (1977), 265-324.

[5] D. Deckard and C. Pearcy, On matrices over the ring of continuous complex-valued functions on a Stonian space, Proc. Amer. Math. Soc. 14 (1963), 322-328.

[6] - - -, On continuous matrix-valued functions on a Stonian space, Pacific J. Math. 14 (1964), 857-869.

[7] C. Foias, C. Pearcy and L. Smith, Weak orbit-transitivity on Hilbert space, Acta Sci. Math. (Szeged) 76 (2010), 17-26.

[8] T. Hoover, Hyperinvariant subspaces for n-normal operators, ibid. 32 (1971), 109119.

[9] I. B. Jung, E. Ko and C. Pearcy, Some nonhypertransitive operators, Pacific J. Math. 220 (2005), 329-340.

[10] C. Pearcy, A complete set of unitary invariants for operators generating finite $W^{*}$ algebras of type I, ibid. 12 (1962), 1405-1417.

[11] - On unitary equivalence of matrices over the ring of continuous complex-valued functions on a Stonian space, Canad. J. Math. 15 (1963), 323-331.

[12] -, Some Recent Developments in Operator Theory, CBMS Reg. Conf. Ser. Math. 36, Amer. Math. Soc., Providence, RI, 1978.

[13] C. J. Read, The invariant subspace problem for a class of Banach spaces. II. Hypercyclic operators, Israel J. Math. 63 (1988), 1-40.

Carl Pearcy, Lidia Smith

Department of Mathematics

Texas A\&M University

College Station, TX 77843, U.S.A.

E-mail: pearcy@math.tamu.edu lsmith@math.tamu.edu

Received April 27, 2008

Revised version August 18, 2009 